\title{
Vortex Expulsion by a Zonal Coastal Jet on a Transverse Canyon
}

\author{
Laurent M. Chérubin and Xavier J. Carton \\ SHOM/CMO, Brest, France \\ David G. Dritschel \\ DAMTP, University of Cambridge, Cambridge, UK
}

\begin{abstract}
Recent observations of subsurface-intensified, alongshore slope-currents have shown vortex formation over a transverse canyon, in the Gulf of Cadiz. To analyze this process, we idealize this situation to a zonal coastal jet, with piecewise-constant potential-vorticity, flowing over a meridionally sloping bottom. We analytically calculate the linear barotropic and baroclinic stability of the flow in the quasi-geostrophic framework (in the absence of the canyon). Several physical and geometrical cases are considered. The effect of an additional transverse canyon is simulated numerically using the two-dimensional contour-surgery code. It is shown that

(a) a double strip of vorticity is linearly unstable and a very shallow canyon is sufficient to provoke wave growth on the vorticity interfaces; these waves nonlinearly saturate into a dipolar vortex;

(b) a single active vorticity region is linearly stable, but a deep enough canyon can trigger various nonlinear responses (waves, filaments, vortex detachment, turbulence).

The relevance of such an idealized model to real oceanic cases is finally discussed, its shortcomings are highlighted, and possible improvements are suggested for future work.
\end{abstract}

\section{Motivation for the study}

The Mediterranean Sea is an evaporation basin forming warm and salty water masses. An exchange flow occurs at the Strait of Gibraltar, with eastward-flowing, cold but fresh North Atlantic (Central) water at the surface, and westward-flowing, warm and salty, Mediterranean water beneath it. This latter water plunges into the Gulf of Cadiz to reach the local ocean bottom (the continental slope), and veers north under the influence of the Coriolis force. This same force maintains the flow against the slope. The rough ocean bottom topography (as well as differential mixing) induces a bifurcation of this alongshore current into several cores; Fig. 1 synthetically sketches the presence of Mediterranean water (shaded) as measured in the hydrological sections and the orientation of flow given by the currentmeter moorings (numbers). Two main cores follow the $200 \mathrm{~m}$ and $800 \mathrm{~m}$ isobaths, while lateral branches fall off into canyons. West of $8^{\circ} \mathrm{W}$, the two main currents flow zonally (E-W orientation). Around $8^{\circ} 30^{\prime} \mathrm{W}$, they encounter the Portimao canyon, which is oriented N-S (Fig. 1). A vertical cross-section of the current (Fig. 2) shows that the Mediterranean water cores directly flow over or close to the topography, and meander (hence the alternation of velocity direction in Fig. 2, as the section crosses the flow thrice). Close to the canyon, horizontal velocities reach 30 to $50 \mathrm{~cm} / \mathrm{s}$.

Downstream of the Portimao canyon, large anti-cyclonic lenses of Mediterranean water, as well as cyclonic vortices, have been observed in the vicinity of the flow (Swallow [18]; Prater and Sanford 
[16]). Figure 3 shows a north-south cross-section of the flow, with a typical vortex detachment (on the right) from the coastal current. The roughness of the bottom topography is well marked (shaded). The high salinity tongue (above $36 \mathrm{psu}$ ) confirms the presence of Mediterranean water. The vertical velocity profiles indicate that the double-convex lens (right) is anticyclonic, and is accompanied by a cyclonic motion to the north. Usually, such Mediterranean water eddies (or Meddies as these vortices are called) extend between 600 and $1500 \mathrm{~m}$, with radii of 30 to $50 \mathrm{~km}$ (Fig. 3) and rotate rapidly (their relative vorticities can reach $40 \%$ of the planetary vorticity). They later detach from the flow, close to Cape St Vincent, to drift southwest across the North Atlantic Ocean at speeds of $2-3 \mathrm{~cm} / \mathrm{s}$. Owing to their long lifespan (2 to 8 years) and to their sizeable transport, they strongly modify the temperature and salinity characteristics of the north Atlantic Ocean at depths close to $1000 \mathrm{~m}$.

The stability of zonal coastal flows has been previously addressed in the context of surface jets, often bearing applications to the Algerian current (Killworth and Stern [10], Kubokawa [12], Paldor and Ghil [15], Barth [2]). But the specificities of the Mediterranean outflow are the strong influence of the bottom topography along which it flows, the role of the ambient stratification (via the current's mid-depth immersion), and finally its frontal nature and large heat and salt anomalies (these latter two effects are not taken into account in the present study). To date, several studies have focused on the internal flow structure of the Mediterranean water cores, from the strait downstream, by dint of hydraulic models (Smith [17], O'Neil [14]). Analytical, numerical and experimental work have evaluated the various forms of evolution of a coastal flow over a canyon or a bank (Nof [13], Gjevik and Moe [8], Hugues et al. [9], Klinck [11], Folkard and Davies [7]). Few of these studies dealt with the conjugate aspects of instability, stratification and the influence of bottom topography.

In the present study, we use the simple, but powerful framework of quasi-geostrophy to separately evaluate the role of each physical and geometrical parameter of the zonal coastal flow on its stability and on possible eddy generation in the vicinity of a submarine canyon (Section 2). First, we describe several piecewise-constant potential vorticity distributions which correspond to a jetlike flow profile, in the absence or presence of a sloping bottom. Their respective linear stability to normal-mode disturbances is evaluated analytically (Section 3). Secondly, we examine the nonlinear evolution of these flows - in the two-dimensional context only — with the aid of a high-resolution numerical method called "contour surgery" (Section 4). We conclude with a discussion of future work which will take into account nonlinear baroclinic (multi-layer) flows and additional physical processes not considered in this preliminary study (Section 5).

\section{Basic equations and physical cases}

\subsection{The shallow-water equations}

The dynamics of the ocean's interior are dominated by the Earth's rotation and by the ambient (thermohaline) stratification. These render motions layerwise two-dimensional (in the horizontal plane). It is thus usual to represent the ocean as a stack of homogeneous layers, inside each of which the horizontal momentum and the incompressibility equations are vertically integrated (Fig. 4). This set of equations is traditionally dubbed the multilayer shallow-water equations, which are written in the limit of infinite Reynolds number

$$
\begin{gathered}
{\left[\partial_{t}+u_{j} \partial_{x}+v_{j} \partial_{y}\right] u_{j}-\left(f_{0}+\beta y\right) v_{j}=-\partial_{x} p_{j} / \rho_{j}} \\
{\left[\partial_{t}+u_{j} \partial_{x}+v_{j} \partial_{y}\right] v_{j}+\left(f_{0}+\beta y\right) u_{j}=-\partial_{y} p_{j} / \rho_{j}} \\
{\left[\partial_{t}+u_{j} \partial_{x}+v_{j} \partial_{y}\right] h_{j}+h_{j}\left(\partial_{x} u_{j}+\partial_{y} v_{j}\right)=0 .}
\end{gathered}
$$


where $j=1, \ldots, N$ is the layer index, $u_{j}, v_{j}, p_{j}, h_{j}, \rho_{j}$ are respectively the horizontal velocity components, the pressure, thickness and density for each layer, which all but density depend on $x$, $y, t$.

The layer thickness can be expressed as $h_{j}=H_{j}+\xi_{j-1 / 2}-\xi_{j+1 / 2}$, where $H_{j}$ is the layer thickness at rest, and $\xi_{j-1 / 2}(x, y, t)$ the instantaneous, local elevation of the density interface between layer $j-1$ and $j$. With the further assumptions of a rigid-lid at the ocean surface and irregular bottom topography, we have $\xi_{1 / 2}=0$ and $\xi_{N+1 / 2}=h_{B}(x, y)$. With the rigid-lid assumption, the surface pressure $p_{1}$ becomes an independent variable. In each layer, the pressure $p_{j}$ is related to $p_{1}$ and to the interface deviations $\xi_{j+1 / 2}$ by hydrostatic balance: $p_{j}=p_{1}+\sum_{2}^{j} g\left(\rho_{j}-\rho_{j-1}\right) \xi_{j-1 / 2}$, where $g$ is the gravity. By the Boussinesq approximation, the local density dividing the pressure term in the momentum equations can be approximated by an average density $\rho_{0}$. Thus reduced gravities are defined as $g_{j-1 / 2}^{\prime}=g\left(\rho_{j}-\rho_{j-1}\right) / \rho_{0}$.

The planetary vorticity, or Coriolis parameter, is $f=2 \Omega \sin \theta$, where $\Omega$ is the Earth's rotation rate and $\theta$ the local latitude on its surface. The Coriolis parameter is usually expanded linearly in the meridional direction $y$ as $f=f_{0}+\beta y$ around a latitude of reference, if the meridional extension of the flow domain is small compared to the Earth radius. In most cases here, we will assume $\beta \approx 0$ since our zonal currents are very narrow. Finally, we note that the vertical velocity is defined by $w_{j \pm 1 / 2}=d \xi_{j \pm 1 / 2} / d t$ at the density interfaces.

It is now straightforward to take the curl of the momentum equations to eliminate the pressure, and to substitute the horizontal velocity divergence from the incompressibility equation. This yields the conservation of potential vorticity:

$$
\left[\partial_{t}+u_{j} \partial_{x}+v_{j} \partial_{y}\right] \Pi_{j}=0, \quad \Pi_{j}=\frac{\zeta_{j}+f}{h_{j}}, \quad \zeta_{j}=\partial_{x} v_{j}-\partial_{y} u_{j},
$$

the latter term being the relative vorticity. It must be noted that, in the case of parallel (here zonal) currents, a simple diagnostic relation, the geostrophic balance, holds between velocity and pressure. Indeed, if $u_{j}=u_{j}(y), v_{j}=0, h_{j}=h_{j}(y), p_{j}=p_{j}(y)$ (whatever $j$ ),

$$
\left(f_{0}+\beta y\right) u_{j}=-\frac{1}{\rho_{0}} \frac{d p_{j}}{d y} .
$$

\subsection{The quasi-geostrophic equations}

With the further assumptions that the relative acceleration be small compared to the Coriolis acceleration (small Rossby number Ro), that the buoyancy forces be comparable to the Coriolis force (Burger number $B u$ of order unity) - hence small interface elevations - and that the $\beta$ effect be small, the flow is at zeroth order in Ro, two-dimensional, non-divergent and in geostrophic balance. The flow derives from a streamfunction $u_{j}=-\partial_{y} \psi_{j}, v_{j}=\partial_{x} \psi_{j}$, which is related to pressure by $\psi_{j}=p_{j} / \rho_{0} f_{0}$. The conservation of potential vorticity then becomes:

$$
\left[\partial_{t}+J\left(\psi_{j}, \cdot\right)\right] \Pi_{j}=0
$$

where $J(a, b)=\partial_{x} a \partial_{y} b-\partial_{y} a \partial_{x} b$ is the antisymmetric Jacobian operator, and

$$
\Pi_{j}=\nabla^{2} \psi_{j}+F_{j, j+1}\left(\psi_{j+1}-\psi_{j}\right)+F_{j, j-1}\left(\psi_{j-1}-\psi_{j}\right)
$$

for an intermediate layer; for the top and bottom layers, the equations simplify accordingly. The coefficients noted $F_{j, j+1}=f_{0}^{2} /\left(g_{j+1 / 2}^{\prime} H_{j}\right)$ and $F_{j, j-1}=f_{0}^{2} /\left(g_{j-1 / 2}^{\prime} H_{j}\right)$ couple adjacent layers. The Laplacian operator is purely horizontal: $\nabla^{2}=\partial_{x}^{2}+\partial_{y}^{2}$. 
A simpler relation between potential vorticity and streamfunction can be obtained when the flow is projected onto vertical modes instead of layers (the expression of each modal quantity, labelled by $m$, is a linear combination of layerwise quantities):

$$
\Pi_{m}=\nabla^{2} \psi_{m}-\lambda_{m}^{2} \psi_{m}
$$

Now the streamfunction can be directly computed from the potential vorticity by a kernel integral, with a Green function depending on the vertical structure of the flow:

$$
\psi_{m}(\mathbf{r})=\iint \Pi_{m}\left(\mathbf{r}^{\prime}\right) G\left(\mathbf{r}-\mathbf{r}^{\prime}, \lambda_{m}\right) d x^{\prime} d y^{\prime} .
$$

Furthermore, if the flow is composed of several horizontal domains $\mathcal{D}_{l}$, inside each of which potential vorticity is constant,

$$
\Pi_{m}(\mathbf{r})=\sum_{l} \Pi_{m l} \Xi\left(\mathcal{D}_{l}\right),
$$

where $\Xi$ is the "characteristic function" (unity inside $\mathcal{D}_{l}$ and zero outside), then the horizontal velocity can be computed from Eq. 4 as:

$$
\mathbf{u}_{m}(\mathbf{r})=-\sum_{l} \Pi_{m l} \oint G\left(\mathbf{r}-\mathbf{r}^{\prime}, \lambda_{m}\right) d \mathbf{r}^{\prime}
$$

The Green function $G$ is $(2 \pi)^{-1} \log \left|\mathbf{r}-\mathbf{r}^{\prime}\right|$ for $m=0\left(\lambda_{0}=0\right)$ - this being the "barotropic" or two-dimensional mode - else it is $-(2 \pi)^{-1} K_{0}\left(\lambda_{m}\left|\mathbf{r}-\mathbf{r}^{\prime}\right|\right)$ for $m>0\left(\lambda_{m}>0\right)$ - these being the "baroclinic" or three-dimensional modes. A numerical code can thus be built upon this formula (Dritschel and Saravanan [6]) to simulate the evolution of any potential vorticity field purely in terms of contours of potential vorticity discontinuity. The original two-dimensional method was called "contour dynamics" (Zabusky et al. [19]). A sophisticated version of this method, including an automatic means for contour reconnection at small scales, "contour surgery" (Dritschel [5]), is employed in the present study.

\subsection{Perturbation equations}

The mean state is a parallel zonal flow $\bar{\psi}_{j}(y), \bar{u}_{j}(y)$, with piecewise-constant potential vorticity $\bar{\Pi}_{j}(y)=\sum_{l} \Pi_{j l} \Xi\left(0 \leq y<Y_{j l}\right)$, where $y=Y_{j l}$ (layer index $j$, interface number $l$ ) are the stationary potential-vorticity interfaces. Since any regular disturbance can be decomposed into normal-mode perturbations, we choose to perturb the flow with a monochromatic-wave interface displacement $y=Y_{j l}+\eta_{j l} \exp (i k[x-c t])$, with $\left|\eta_{j l}\right|<<\left|Y_{j l}\right|$. This results in streamfunction anomalies of the form $\psi_{j}^{\prime}(x, y, t)=\phi_{j}(y) \exp (i k[x-c t])$. Equation 4 then gives the relation between $\phi_{j}\left(Y_{j l}\right)$ and $\eta_{j l}$ in the linear regime:

$$
\phi_{j}\left(Y_{j l}\right)=\sum_{n p} \Pi_{n p} \eta_{n p} \int_{-\infty}^{+\infty} G_{j n}\left(\left(x-x^{\prime}\right)^{2}+\left(Y_{j l}-Y_{n p}\right)^{2}\right) \exp \left(i k\left[x^{\prime}-x\right]\right) d x^{\prime}
$$

where the sum is over layers and vorticity interfaces, with $G_{j n}$, the layerwise Green function, a linear combination of the modal Green fuction previously explicitly stated. We symbolically write the latter integral $G_{j n}^{l p}$. Taking into account the image vorticity by the wall, we can write the linearized equations of motion, for the potential vorticity interfaces:

$$
\left(\partial_{t}+\bar{u}_{j}\left(Y_{j l}\right) \partial_{x}\right)\left(Y_{j l}+\eta_{j l} \exp (i k[x-c t])\right)=v_{j l}^{\prime}=\partial_{x}\left(\phi_{j}\left(Y_{j l}\right) \exp (i k[x-c t])\right)
$$


which reduces to a simple eigenvalue problem

$$
\bar{u}_{j}\left(Y_{j l}\right) \eta_{j l}+\sum_{n p} G_{j n}^{l p} \Pi_{n p} \eta_{n p}=c \eta_{j l}
$$

Now, we have all the necessary elements to evaluate the growth rate $k c_{i}$ for various alongshore jets.

\section{$3 \quad$ Linear stability results}

\subsection{Physical and geometrical set-up; linear stability}

\section{Case A: one-layer single jet case, with two vorticity strips}

In two-dimensional flows, a zonal jet-like profile can be obtained by juxtaposing two strips of vorticity along the coast, with respective vorticities $Q_{1}, Q_{2}$ and $Q_{3}$ outside (Fig. 5). If we assume that the bottom slopes down from north to south (constant slope $s$ ), we can derive the mean flow by $Q_{l}=-d \bar{u}_{l} / d y+s y$ in the three regions $(l=1,2,3)$. We have to assume that the flow is bounded to the south by a second wall (channel configuration), since otherwise, the velocity shear would diverge at infinity (south) due to infinite bottom depth. The resulting flow is:

$$
\begin{gathered}
\bar{u}_{1}(y)=-Q_{1} y+s y^{2} / 2, \quad-b<y<0 ; \\
\bar{u}_{2}(y)=-1-Q_{2}(y+b)+s\left(y^{2}-b^{2}\right) / 2, \quad-1<y<-b ; \\
\bar{u}_{3}(y)=-Q_{3}(y+1)+s\left(y^{2}-1\right) / 2, \quad-L<y<-1 .
\end{gathered}
$$

with $\bar{u}_{1}(0)=0, \bar{u}_{2}(-b)=-1, \bar{u}_{3}(-1)=0$. Continuity of $\bar{u}$ implies $Q_{1}=-1 / b+s b / 2, Q_{2}=$ $1 /(1-b)-s(1+b) / 2$; with the continuity of bottom topography, and zero relative vorticity at the outer boundary, $Q_{3}=-s$. In our previous notations (Eq. 5), we have $\Pi_{1}=Q_{2}-Q_{1}, \Pi_{2}=Q_{3}-Q_{2}$.

The interface displacement equations can now be written:

$$
\begin{gathered}
(k+\sigma) \eta_{1}=\frac{\Pi_{1} \eta_{1}}{2} \alpha-\frac{\Pi_{2} \eta_{2}}{2} \beta \\
\sigma \eta_{2}=\frac{\Pi_{2} \eta_{2}}{2} \gamma-\frac{\Pi_{1} \eta_{1}}{2} \beta
\end{gathered}
$$

with $\alpha=\exp (-2 k b)-1, \beta=\exp (-k[b+1])-\exp (-k[1-b]), \gamma=\exp (-2 k)-1, \sigma=k c_{i}$. For non-zero interface displacements $\eta_{l}$, we need to set the determinant of these equations to zero. It yields a second-degree equation in $\sigma$ for which the discrimant is $\Delta=\left[\Pi_{1} \alpha / 2-\Pi_{2} \gamma / 2-k\right]^{2}+\beta^{2} \Pi_{1} \Pi_{2}$ which has to be negative for instability to be present. The values of the growth rate $\sigma=k c_{i}=k \sqrt{\Delta}$ are given on Fig. 6 in the $k, b$ plane for $s=0$ and $s \neq 0$. We observe that (a) whatever $b$, there is nearly always one unstable wavenumber $k$ in the flat-bottom case, (b) the sloping bottom tends to destabilize the flow (it acts here as an opposite $\beta$-effect, since the flow is two-dimensional).

\section{Case B: one- and two-layer, single-jet case, with one vorticity strip}

A coastal jet-like profile can be obtained by considering a single vorticity strip, including a continuously sloping bottom topography (Fig. 7a). We have $Q_{l}=-d \bar{u}_{l} / d y+\left(f_{0} h_{b} / H\right) \exp \left(y / L_{b}\right)$. We can set $f_{0} h_{b} / H=1$ and $L_{b}=1$ as time and space scales. With $\bar{u}_{0}=\bar{u}(0)$, the velocity profile is then:

$$
\begin{gathered}
\bar{u}=\bar{u}_{0}-Q_{1} y+\exp (-y)-1, \quad-a<y<0 \\
\bar{u}=\bar{u}_{0}+Q_{1} a-Q_{2}(y+a)+\exp (y)-\exp (-a), \quad-\infty<y<-a .
\end{gathered}
$$


We can set $Q_{2}=0$ without modifying the problem. The velocity maximum is located at $y=$ $\log \left(-Q_{1}\right)$. We want $d \bar{u} / d y(0)<0$, which implies $Q_{1}<-1$ and $-a<\log \left(-Q_{1}\right)<0$ or $Q_{1}<$ $-\exp (-a)$, which is more stringent than the previous condition. The instability condition is then written $k(\bar{u}(-a)-c)-\left(Q_{1} / 2\right)(\exp (2 k a)-1)=0$, and the dispersion relation is thus:

$$
\sigma=-\left(Q_{1} / 2\right)((\exp (2 k a)-1)+k \bar{u}(-a)
$$

Therefore, the flow is linearly stable. Simple calculations can be performed to anticipate the jet behavior over the canyon: if we set $f_{0} h_{b} / H=f(x)$ and compute the velocity and the (conserved) transport $\left(\int u d y\right)$, we can show that the flow has to narrow over the canyon. An instability condition states that the critical point has to reach the jet boundary. This occurs only where the jet boundary joins the coast, but there the transport cannot be conserved since the jet width vanishes. This means that linear instability cannot be achieved. Intense nonlinear processes are therefore required for instability to develop. Still, this simple criterion indicates that, if a vortex is to detach from the jet, the jet must strongly bend towards the coast.

This simple jet configuration can be extended to a stratified (two-layer) fluid, where the current flows in the lower layer. First, we can assume that the upper layer is infinitely thick and at rest (this is dubbed the one-and-a-half layer model, Fig. 7b). The mean flow can be computed by: $Q_{2 l}=d^{2} \bar{\psi}_{2} / d y^{2}-\lambda_{1}^{2} \bar{\psi}_{2}+\exp (y)$. The mean velocity can be written as:

$$
\begin{gathered}
\bar{u}_{2}=\lambda_{1}\left(B \exp \left(-\lambda_{1} y\right)-A \exp \left(\lambda_{1} y\right)\right)-\exp (y), \quad-a<y<0 \\
\bar{u}_{2}=-\lambda_{1} C \exp \left(\lambda_{1} y\right)-\exp (y), \quad-\infty<y<-a
\end{gathered}
$$

with $A=\left(Q_{21} \exp \left(-\lambda_{1} a\right) / 2 \lambda_{1}^{2}\right)-\left(\bar{u}_{0}+1 / \lambda_{1}\right), B=Q_{21} \exp \left(-\lambda_{1} a\right) / 2 \lambda_{1}^{2}, C=-\left(Q_{21} \sinh \left(\lambda_{1} a\right) / \lambda_{1}^{2}\right)-$ $\left(\bar{u}_{0}+1 / \lambda_{1}\right)$, with the continuity conditions. Since

$$
\psi_{2}^{\prime}(x, y, t)=-\frac{Q \delta Y_{21}}{4 \pi K_{\lambda}} \exp (i k[x-c t])\left[\exp \left(-K_{\lambda}|y+a|-\exp \left(-K_{\lambda}|y-a|\right)\right],\right.
$$

we can write the dispersion relation as:

$$
\sigma=k\left[\bar{u}(-a)-\frac{Q}{2 K_{\lambda}}\left(1-\exp \left(-2 K_{\lambda} a\right)\right)\right]
$$

thus proving that the flow is linearly stable. (This is of course always true for monotonic distributions of potential vorticity, even to finite amplitude - Dritschel [4].)

A supplementary degree of freedom can be added to the problem when we consider two active layers of fluid (having finite upper-layer depth) with the current still flowing on the topography in the lower layer (Fig. 7c). This new problem is formally the addition of the former two. Analytical details will not be given here for lack of space, but the mathematically-oriented reader is referred to Chérubin [3] where the proof of linear stability is extensively provided.

\section{Case C: Two-layer, multiple jet case}

Finally, the two cores of Mediterranean water flowing parallel to each other can be idealized in a twolayer model as two adjacent alongshore jets, vertically separated (Fig. 7d). Again, the mathematical details can be found in Chérubin [3]. The algebra is so tedious that the mathematical formula is not extensively indicated; nevertheless, it is applied to the observed values of the physical parameters for the Mediterranean water currents in the Gulf of Cadiz. The result is given in Fig. 8: the growth rate is plotted versus $L_{0} / L$, with $L_{0}$ the lower-layer jet width at rest and $L$ its local and instantaneous value. It shows that, for the flow to be linearly unstable, the bottom current must narrow by a factor 1.2 , when $k=2 \times 10^{-5} \mathrm{~m}^{-1}$. This can occur when the lower jet encounters a submarine canyon. That type of instability occurs for wavenumbers smaller than $k=2 \times 10^{-5} \mathrm{~m}^{-1}$. The instability threshold (the lower-layer jet width) decreases when $k$ increases. 


\subsection{Stationary two-dimensional flow over the canyon}

The first flow-configuration studied in case (B), subsection 3.1, can be extended to include the presence of the canyon with $f_{0} h_{b} / H=f(x)$. We assume moreover that the canyon width (alongshore) is large compared to the jet width. We call $X=\epsilon x$ this stretched coordinate. We thus have $f(x)=h_{c}(X)$. We call $a=a(X)$ the width of the vorticity band over the canyon, with $a=a_{\infty}$ when $X \rightarrow \infty$. We set $Q_{1}=1$ to define a time scale. The horizontal velocity can be decomposed into two parts, one due to the strip of vorticity $\left.u_{c}, v_{c}\right)$ and the other due to the topography $\left(u_{t}, v_{t}\right)$.

First, we compute the part due to the vorticity band, directly and by a kernel integral:

$$
\nabla^{2} \psi_{c}=\frac{d^{2} \psi_{c}}{d y^{2}}+\epsilon^{2} \frac{d^{2} \psi_{c}}{d X^{2}}=1
$$

therefore $u_{c}=u_{c 1}(X)-y$. We also have:

$$
\psi_{c}=\frac{-1}{4 \pi} \int_{0}^{\infty} d y^{\prime} \int_{-\infty}^{\infty} d x^{\prime} H\left(a\left(\epsilon x^{\prime}\right)\right) \log \frac{\left(x-x^{\prime}\right)^{2}+\left(y-y^{\prime}\right)^{2}}{\left(x-x^{\prime}\right)^{2}+\left(y+y^{\prime}\right)^{2}}
$$

with the coastal image vorticity included. We can expand $H\left(a\left(\epsilon x^{\prime}\right)\right)$ around $x$ and insert it into the integral computed for $u_{c}(X, 0)$. This finally leads to $u_{c}(X, 0)=a_{\infty}-a(X)$, since at infinity, the flow is antisymmetric and the velocity vanishes along the wall.

We can now write the motion of the vorticity interface in $X, a(X),\left(u_{c}+u_{t}\right) * d a / d X=\left(v_{c}+v_{t}\right)$. But $v_{c}$ can be expressed as a Hilbert transform of the vorticity interface $a$; this means $v_{c}=$ $c_{r} * d a / d X$, where $c_{r}$ is the phase speed. In the long-wave limit, $c_{r}=(1-\exp (-2 k|a|) / 2 k \approx|a|=-a$. The equation of motion thus simplifies into:

$$
\left(a_{\infty}-a(X)+u_{t}\right) \frac{d a}{d X}=v_{t}
$$

We are now left with the computation of $\psi_{t}, u_{t}, v_{t}$. This can be achieved directly by:

$$
\nabla^{2} \psi_{t}=\partial_{y}^{2} \psi_{t}+\epsilon^{2} \partial_{x}^{2} \psi_{t}=\left[h_{0}^{\infty}-h_{c}(X)\right] \exp (y)
$$

with $h_{0}^{\infty}$ the topography at infinity in $X$. This gives:

$$
\psi_{t}=-h_{0}^{\infty} \exp (y)+b_{0} y+h_{c}(X)\left[\exp (y)-1+c_{0} y\right]
$$

With $h_{c}(X) \rightarrow 0, X \rightarrow \infty$, we have $u_{0}^{\infty}=h_{0}^{\infty}-b_{0}$. The velocity calculation can also be performed by a kernel integral. It yields $u_{t}(X, 0)=-h_{c}(X)$ and thus $c_{0}=0$. The steady form of the total velocity can be written in this long-wave approximation as

$$
u=u_{t}+u_{c}=a_{\infty}-a-y+u_{0}^{\infty}+h_{0}^{\infty}[\exp (y)-1]-h_{c}(X) \exp (y) .
$$

In a stationary configuration, the vorticity interface is a streamline. We want to define its position everywhere, and especially on the canyon as an initial condition for the QG model. We thus have to compute the transport $\psi$ :

$$
\psi(X, y)=\left(a_{\infty}-a\right) y-y^{2} / 2-h_{0}^{\infty} \exp (y)+\left(h_{0}^{\infty}-u_{0}^{\infty}\right) y+h_{c}(X)[\exp (y)-1+y]
$$

which is a constant for $y=-a(X)$ with $a(X) \rightarrow a_{\infty}, X \rightarrow \pm \infty$. Hence

$$
\begin{gathered}
\left(a_{\infty}-a\right) a-\frac{a^{2}}{2}-h_{0}^{\infty} \exp (a)+\left(h_{0}^{\infty}-u_{0}^{\infty}\right) a+h_{c}(X)[\exp (a)-1+a]= \\
-\frac{a_{\infty}^{2}}{2}-h_{0}^{\infty} \exp \left(a_{\infty}\right)+\left(h_{0}^{\infty}-u_{0}^{\infty}\right) a_{\infty} .
\end{gathered}
$$

This formula will be used in the following section to initialize the nonlinear numerical contour surgery code. 


\section{The evolution of two-dimensional jet flows}

The two-dimensional contour surgery code has first been initialized with the flow given in subsection (3.1.A) with $b=1 / 2, s=0$ (Fig. 8). A Gaussian canyon is located in the middle of the domain perpendicular to the coast and extends all the way across the channel. Both the canyon width and depth have been varied without qualitative change in the flow evolution (only the time-scale of the processes is altered). The time-evolution shows the growth of a meander with a phase shift between the two vorticity interfaces. The initially northernmost negative vorticity region wraps into a pole downstream of the positive vorticity lobe. This indicates a certain recirculation zone and associated closed streamlines close to the coast. To detach from the coast, the fluid particles must slow down when they approach this recirculation zone. This points to a negative vorticity anomaly there. In the positive vorticity strip, the fluid is therefore accelerated, the anomalous bulge is advected downstream and away from the coast, and vorticity does not have time to pile up to a large extent in this outer lobe. But the positive vorticity anomaly growing there also has the effect of accelerating the inner fluid and to reinforce the inner lobe. The angle between the axis of this vorticity dipole and the coast therefore increases with time until its translation velocity is sufficient to leave the jet.

When the dipole trajectory passes downstream, it encounters a bottom topography sloping up. It is therefore squeezed and by conservation of potential vorticity, relative vorticity decreases. Hence the negative pole strengthens and the positive vorticity is advected around it to reach a configuration parallel to the coast, along which it finally propagates (not shown here). This observation supports the recent hypothesis that Mediterranean water eddies may form on the Portimao canyon, but fully detach from the coastal jet only farther downstream, in the vicinity of Cape St Vincent (R.

Käse, priv. comm.). In the real ocean, several factors could account for the preferred survival of the anticyclonic vortex, such as stratification, ageostrophy and the $\beta$-effect, not taken into account here.

A second series of experiments has been performed with the single vorticity strip on an exponentially-sloping bottom described in subsection (3.1.B), still in the two-dimensional case. The position of the vorticity interface was given by Eq. 7, in subsection 3.2. Both the canyon width and depth were varied. For a wide and shallow canyon (Fig. 9a), $w=40 \mathrm{~km}, h_{b}=50 \mathrm{~m}$, the stationary solution remains virtually unchanged. When the canyon becomes narrower $w=30 \mathrm{~km}$, the waves propagates, amplifies and generates shorter wavelength (Fig. 9b); the long-wave approximation is broken. Finally, for a narrow canyon, $w=25 \mathrm{~km}$, the wave amplification results in filament shedding (Fig.9c). When the canyon is deep, $w=35 \mathrm{~km}, h_{b}=150 \mathrm{~m}$, vortices detach from the jet (Fig. 9d). If the canyon is even deeper, many filaments are generated in the vicinity of the jet and prevent any vortex generation by filament roll-up. A turbulent regime is attained (not shown here).

\section{$5 \quad$ Summary and future work}

We have computed analytically the linear stability of various alongshore, zonal jets with several types of stratification. It appears that: (a) a double strip of vorticity, representing a single jet in one layer, or two parallel jets in two different layers, is linearly unstable; the nonlinear evolution of two-dimensional jets ends in dipole expulsion; (b) a jet made of a single strip of vorticity on sloping topography is linearly stable, but the presence of a canyon can disrupt the stationary configuration that exists in the long-wave limit. This results in the formation and expulsion of streamers, vortices or the formation of a turbulent area close to the outer jet boundary. 
From an oceanographic perspective, this study remains highly idealized: it does not take into account baroclinicity and ageostrophy in the nonlinear evolution, the complexity of the topography is oversimplified, and finally the effects of mixing and thermodynamics may be required. Nevertheless, all these factors cannot be considered simultaneously in a quantitative and comprehensive study of flow stability. It seems to us of utmost importance to generalize these preliminary results to stratified flows, and to later add frontal effects by considering the full multi-layer shallow-water framework, described in subsection 2.1. Finally the coupled influences of stratification, topography, ageostrophy and $\beta$-effect will be evaluated.

\section{Acknowledgements}

This research was supported in part by DRET funding of the SHOM/CMO research program "Processus associés aux modèles intermédiaires et régionaux". DGD is supported by the UK Natural Environmental Research Council. We gratefully acknowledge Pr R. Sadourny's hospitality at the Laboratoire de Météorologie Dynamique (Paris) during the course of this work.

\section{References}

[1] Ambar, I. and Howe, M.R., "Observations of the Mediterranean Outflow, II: the Deep Circulation in the Vicinity of the Gulf of Cadiz," Deep-Sea Res., 26 A, pp. 555-568, 1979.

[2] Barth, J.A., "Short-Wavelength Instabilities on Coastal Jets and Fronts," J. Geophys. Res., 99 (C8), pp. 16 095-16 115, 1994.

[3] Chérubin, L., "Instabilités linéaires d'un jet côtier et effet de la bathymétrie: Application à la veine d'eau méditerranéenne," Rapport de DEA d'océanographie, Univ. Aix-Marseille II, 47 p., 1995.

[4] Dritschel, D.G., "Nonlinear Stability Bounds for Inviscid, Two-Dimensional, Parallel or Circular Flows with Monotonic Vorticity, and the Analogous Three-Dimensional Quasi-Geostrophic Flows," J. Fluid Mech., 191, pp. 575-582, 1988.

[5] Dritschel, D.G., "Contour Dynamics and Contour Surgery: Numerical Algorithm for HighResolution Modeling of Vortex Dynamics in Two-Dimensional, Inviscid, Incompressible Flows," Comp. Phys. Rep., 10, pp. 77-146, 1989.

[6] Dritschel, D.G. and Saravanan, R., "Three-Dimensional Quasi-Geostrophic Contour Dynamics, with an Application to Stratospheric Vortex Dynamics," Q. J. Roy. Met. Soc., 120, pp. 12671297, 1994.

[7] Folkard, A. and Davies, P., "An Experimental Study of the Effects of Sloping Topography and Canyons on Boundary Currents in Rotating, Stratified Fluids," Extended abstract 180, 4th International Symposium of Stratified Fluids, Grenoble, 1994.

[8] Gjevik, B. and Moe, H., "Steady and Transient Flows around Banks located near a Shelf Edge," Continental Shelf Res., 14 (12), pp. 1389-1409, 1994.

[9] Hughes, R.L., Ofosu, K.N. and B. Hickey, B., "On the Behavior of Boundary Undercurrents near Canyons," J. Geophys. Res., 95 (C11), pp. 20 259-20 266, 1990. 
[10] Killworth, P.D. and Stern, M.E., "Instabilities on Density-Driven Boundary Currents and Fronts," Geophys. Astrophys. Fluid Dyn., 22, pp. 1-28, 1982.

[11] Klinck, J.M., "The Influence of a Narrow Transverse Canyon on Initially Geostrophic Flows," J. Geophys. Res., 93, pp. 509-515, 1988.

[12] Kubokawa, A., "Instability and Nonlinear Evolution of a Density-Driven Coastal Current with a Surface Front in a Two-Layer Ocean," Geophys. Astrophys. Fluid Dyn., 40, pp. 195-223, 1988.

[13] Nof, D., "The Influence of Varying Bathymetry on Inertial Boundary Currents," Tellus, 32, pp. 284-295, 1980.

[14] O'Neil-Baringer, M., Mixing and Dynamics of the Mediterranean Outflow, PhD thesis, MITWHOI, WHOI-93-52, 244 p., 1993.

[15] Paldor, N. and Ghil, M., "Shortwave Instabilities of Coastal Currents," Geophys. Astrophys. Fluid Dyn., 58, pp. 225-241, 1990.

[16] Prater, M.D. and Sanford, T.B., "A Meddy off Cape St. Vincent. Part I: Description," J. Phys. Oceanogr., 24, pp. 1572-1586, 1994.

[17] Smith, P.C., "A Streamtube Model for Bottom Boundary Currents," Deep-Sea Res., 22, pp. 853-873, 1975.

[18] Swallow, J.C., “A Deep Eddy off Cape St Vincent,” Deep-Sea Res., suppl. to vol. 16, pp. 285-295, 1969.

[19] Zabusky, N.J., Hughes, M.H. and Roberts, K.V., "Contour Dynamics for the Euler Equation in Two Dimensions," J. Comp. Phys., 30, pp. 96-106, 1979.

[20] Zenk, W., "On the Mediterranean Outflow West of Gibraltar," Meteor-Forsch. Ergebnis., Reihe A (16), pp. 23-34, 1975. 


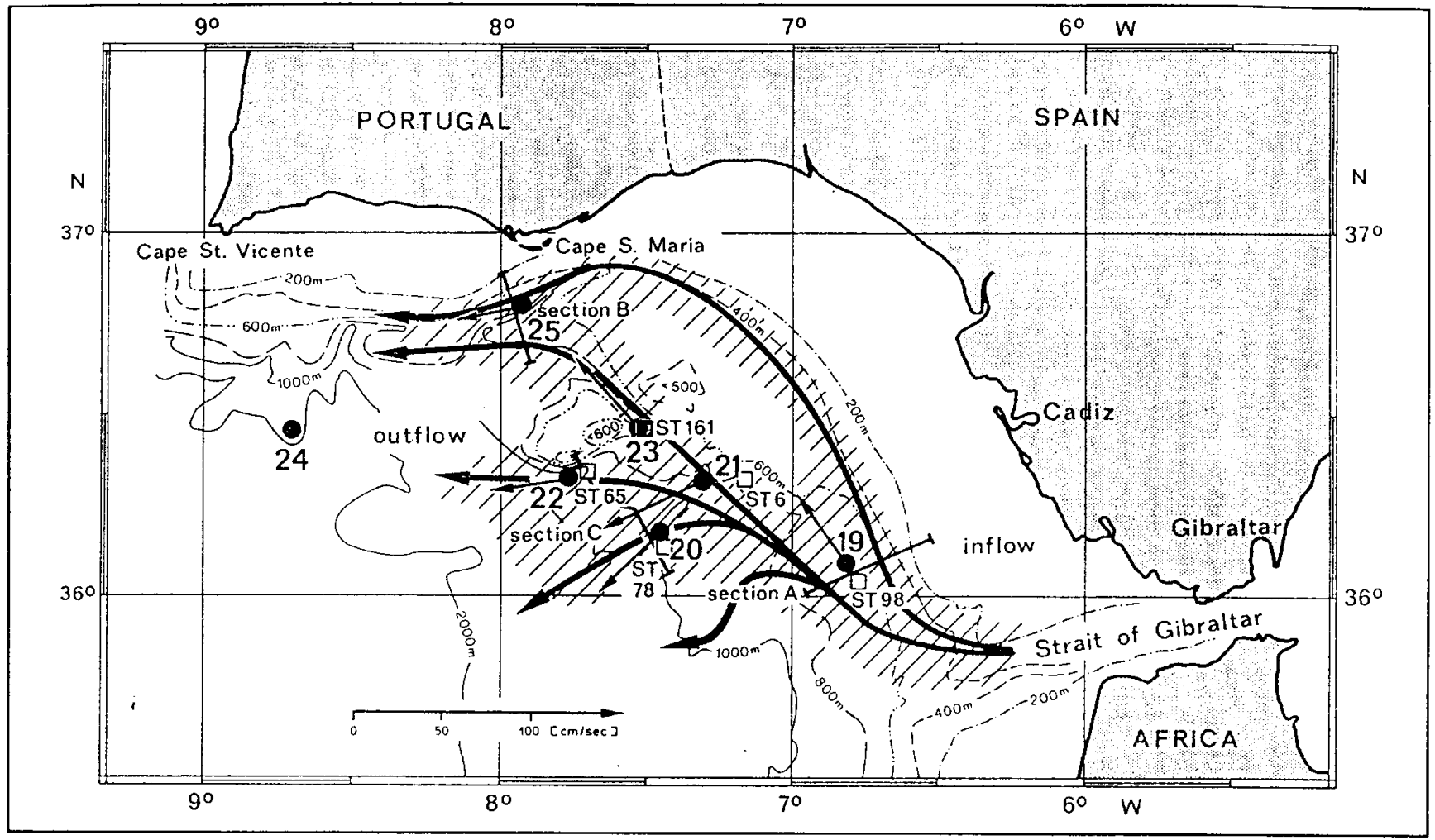

Figure 1: Distribution of the Mediterranean water undercurrent in the gulf of Cadiz from hydrological measurements; horizontal velocities are indicated at the currentmeter moorings (from Zenk $[20])$. 


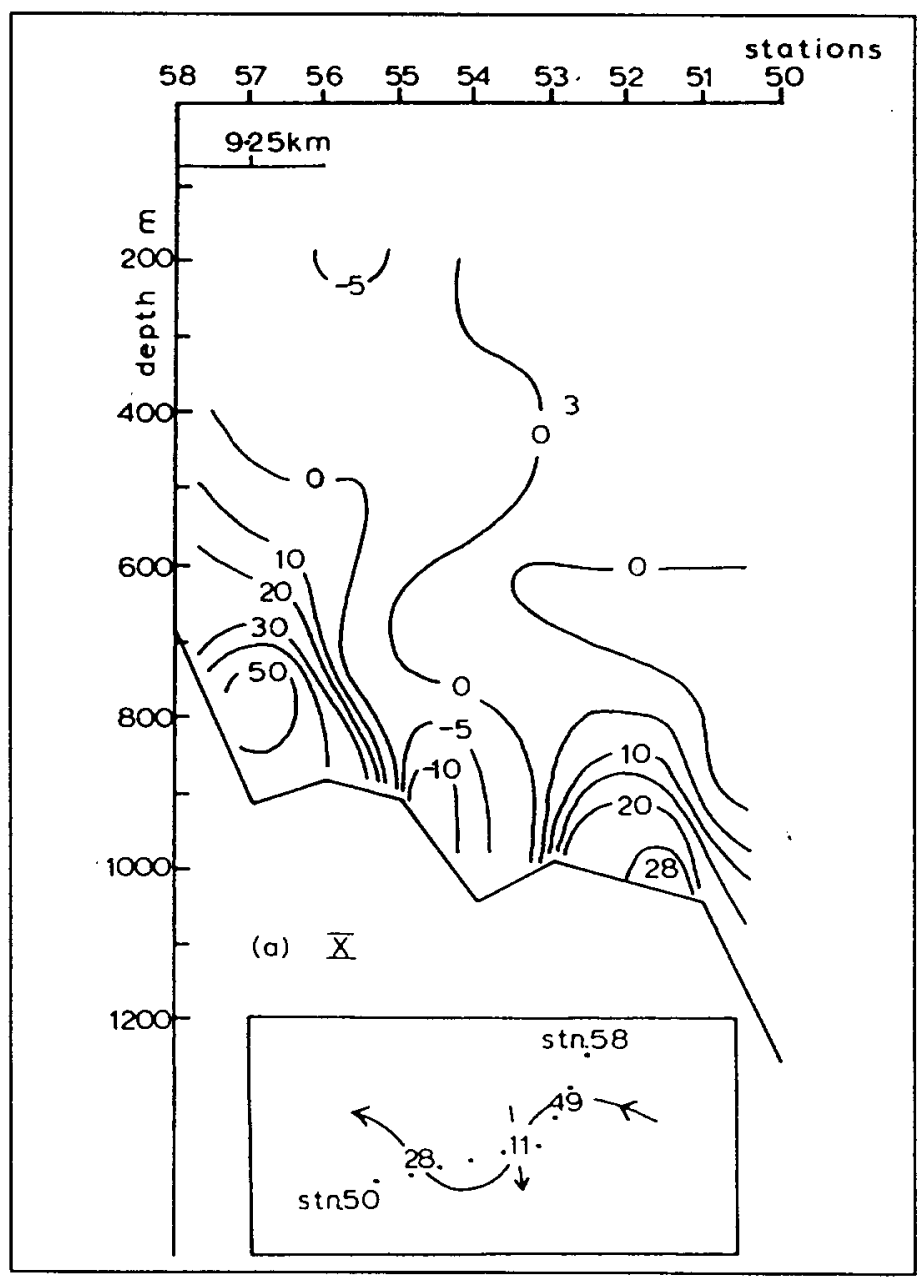

Figure 2: Vertical velocity cross-sections of a meander of the Mediterranean water outflow in the Gulf of Cadiz (from Ambar and Howe [1]). 


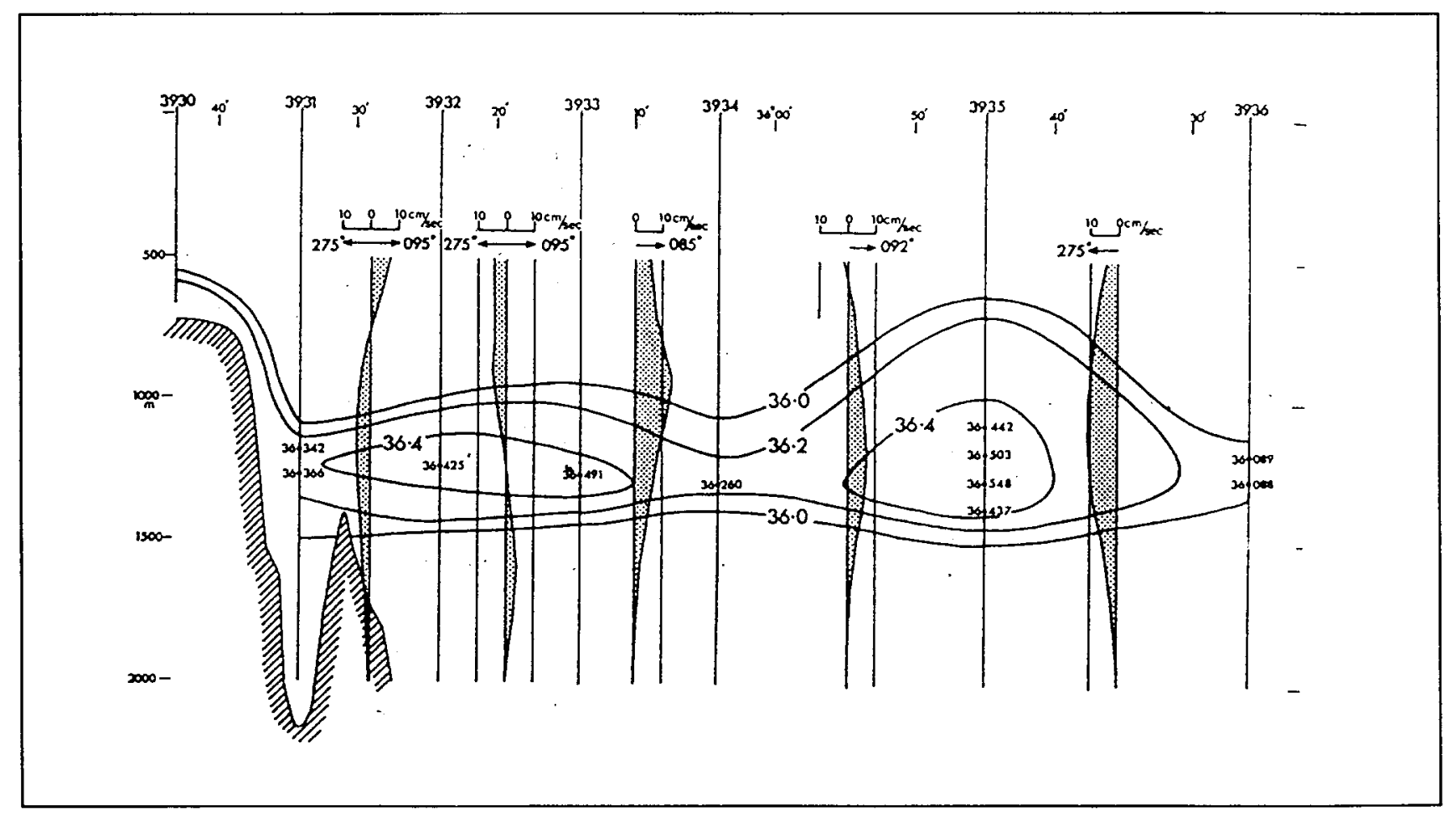

Figure 3: Isohalines of a north-south hydrology section at $8^{\circ} 50^{\prime} W$ showing meddy detachment from the coastal flow; vertical velocity profiles are given at various locations (from Swallow [18]).

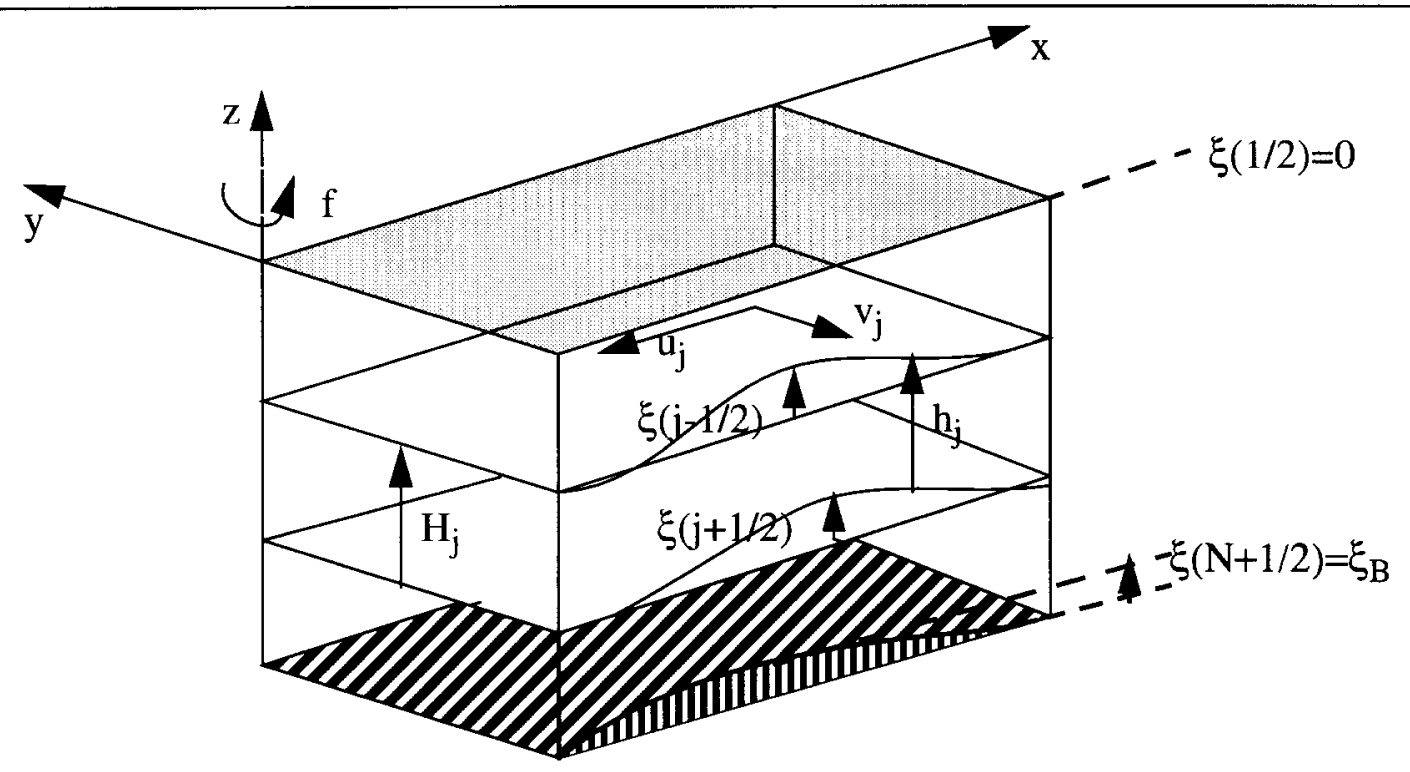

Figure 4: Schematic representation of a layerwise-stratified ocean flow with bottom topography. 

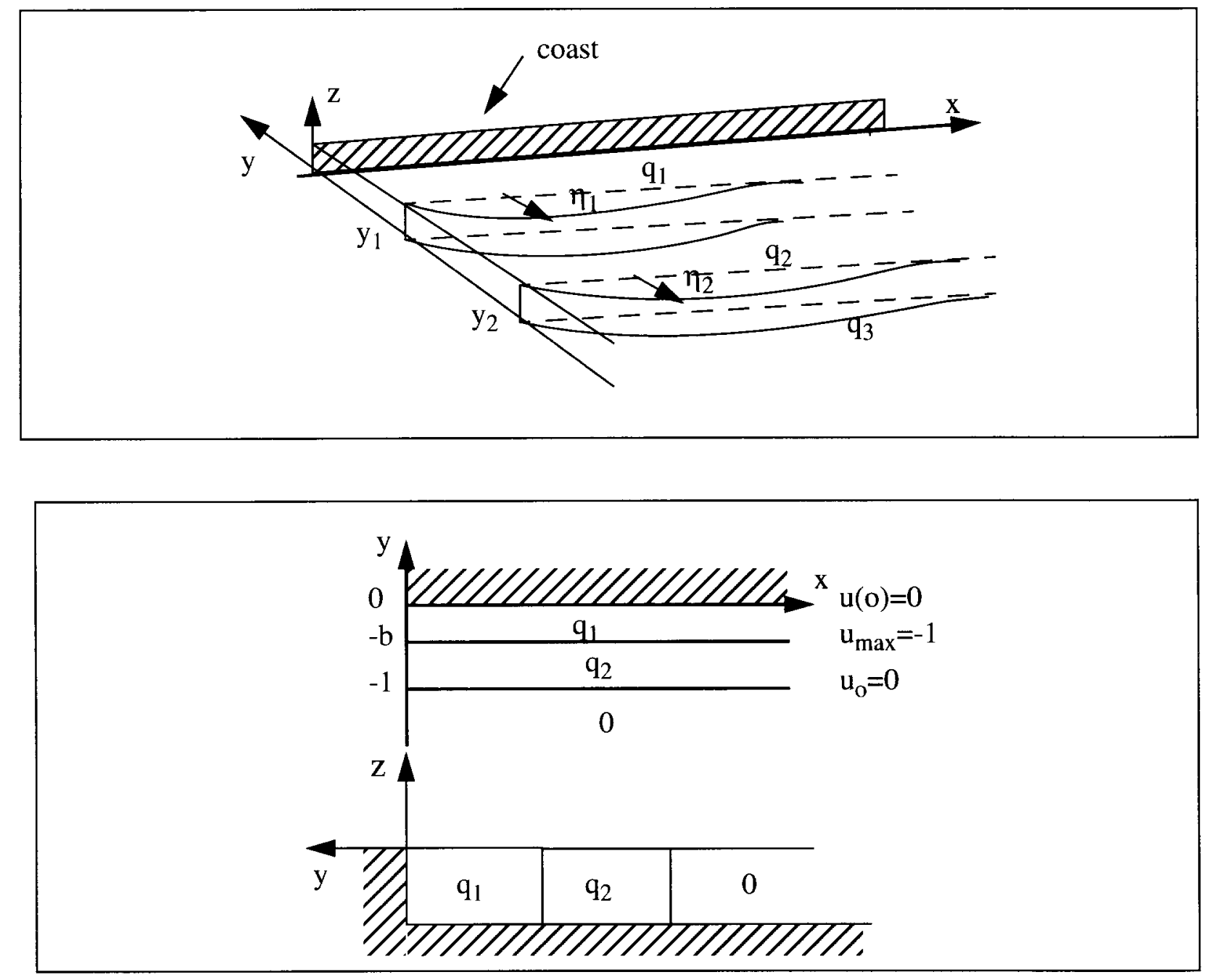

Figure 5: Two-dimensional jet profile made of two vorticity strips. A perspective, plane view and vertical cross-section are shown (a sloping bottom can be added). 


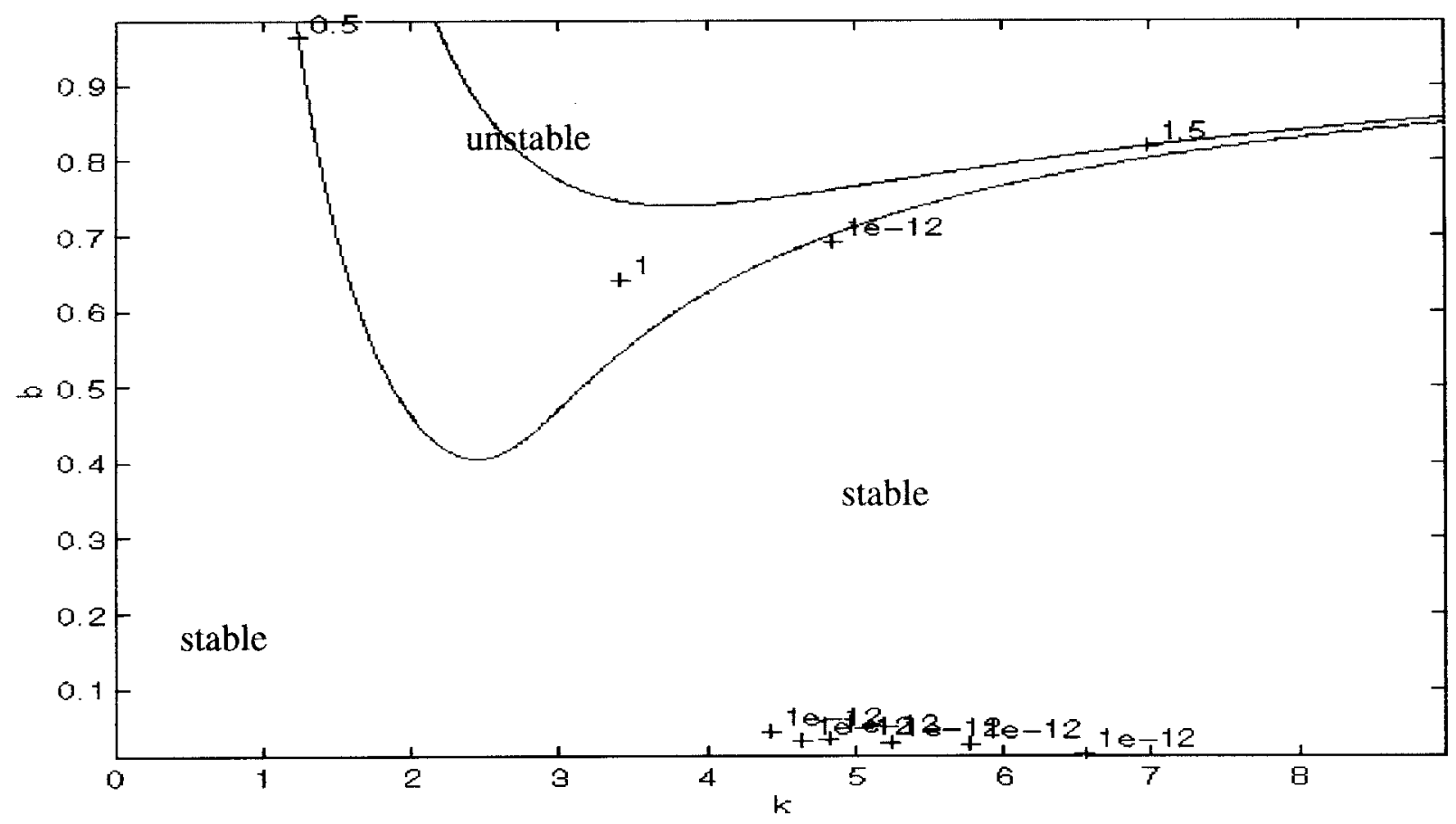

Flat bottom.

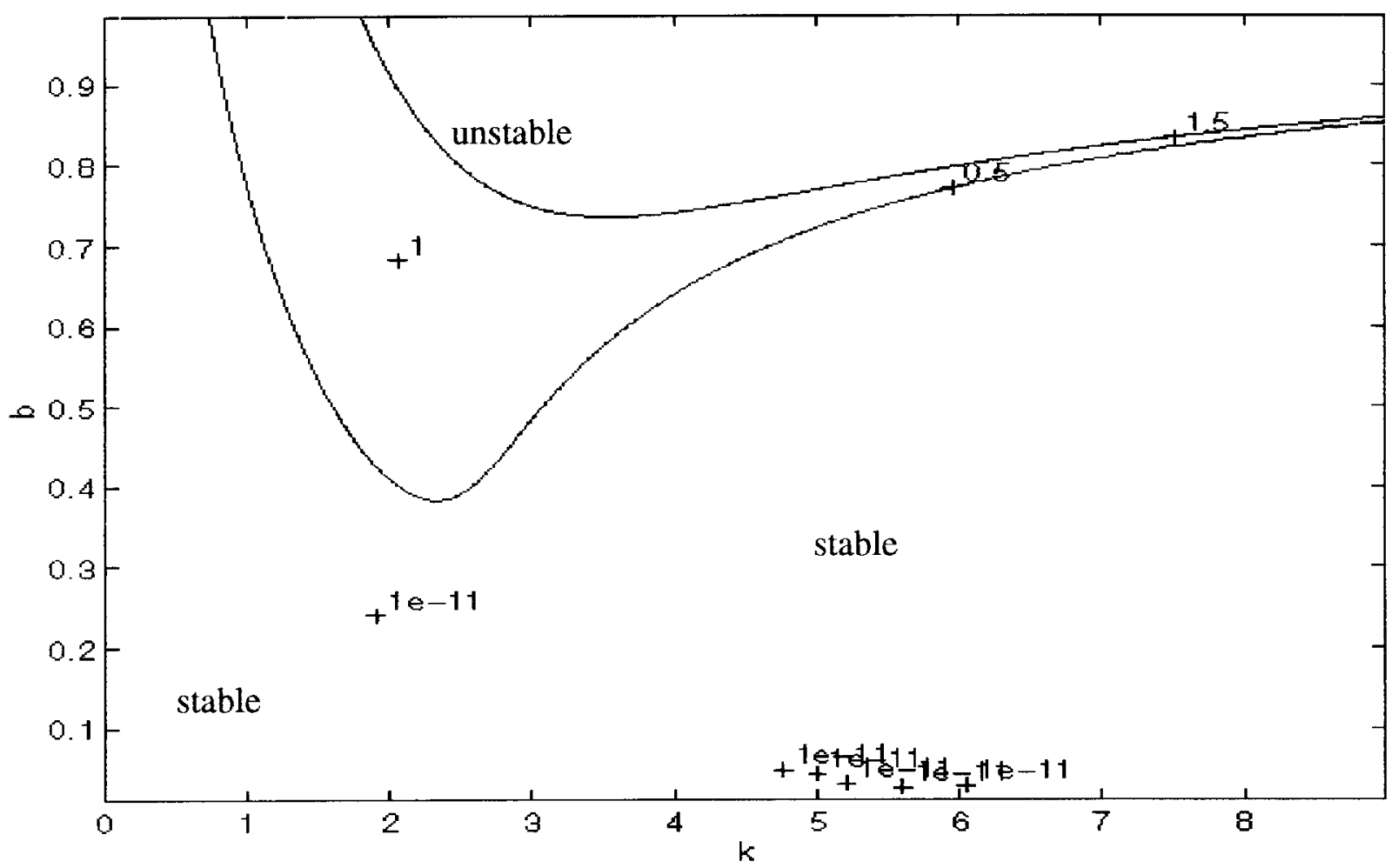

Linearly sloping bottom.

Figure 6: Stability of the flow configuration shown on Fig. 5. 


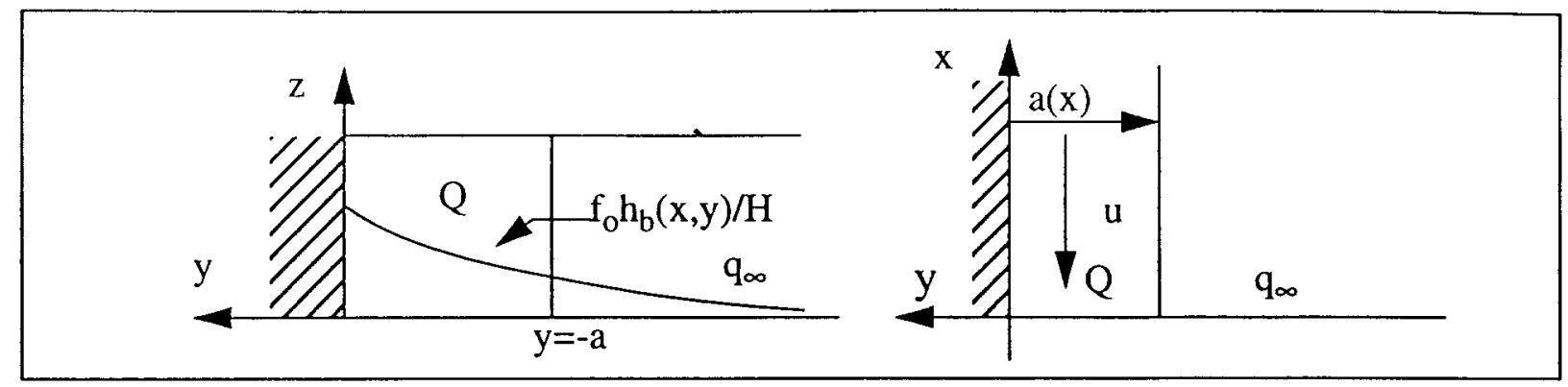

(a)

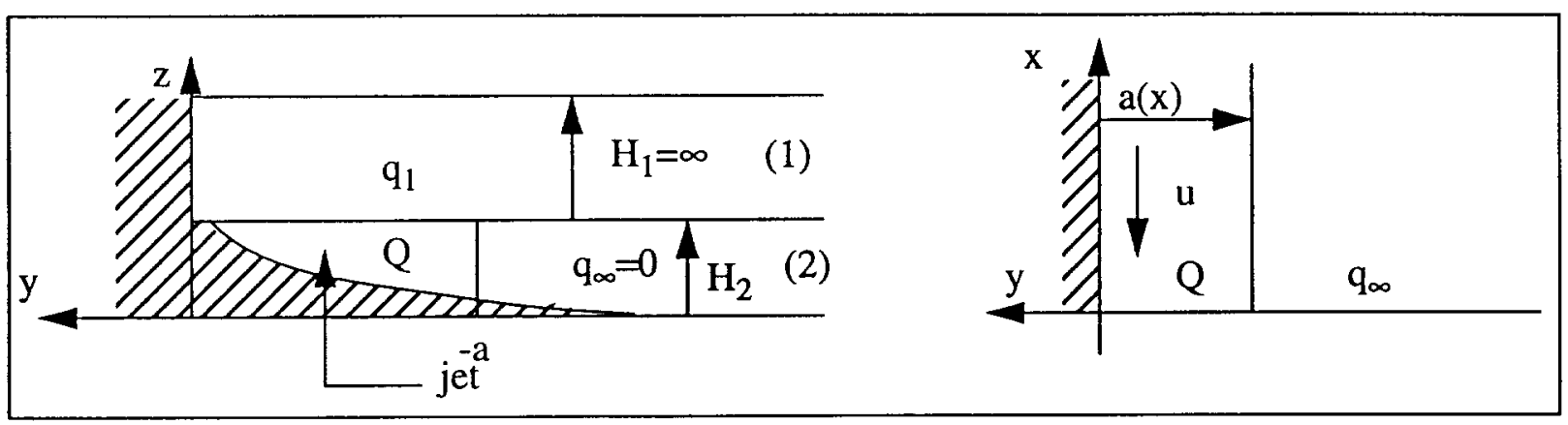

(b)

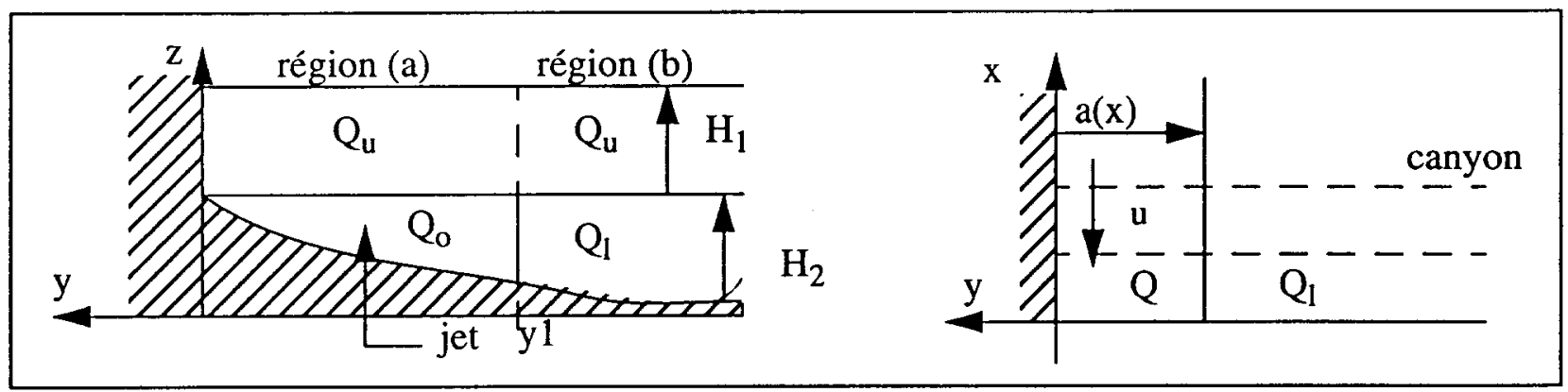

(c)

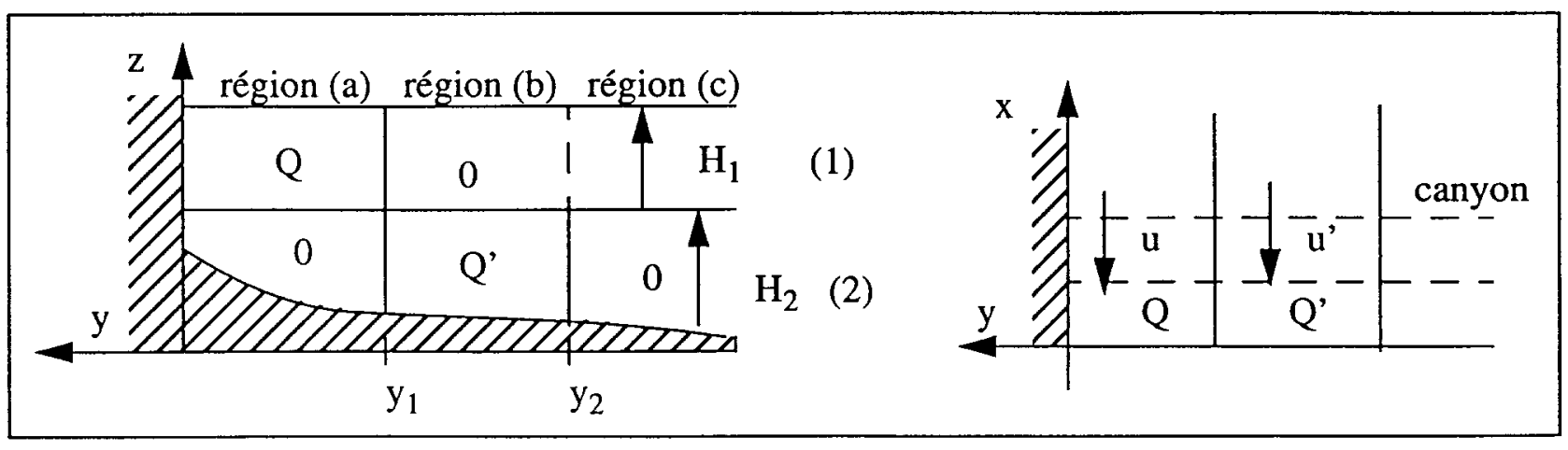

(d)

Figure 7: Top views and cross-sections of (a) a two-dimensional jet obtained by a single vorticity strip flowing over an exponantially-decaying slope; (b) a one-and-a-half equivalent form of 7(a); (c) a two-layer form of 7(a); (d) a two-layer multi-jet flow with two potential-vorticity strips. 


\begin{tabular}{|l|l|l|}
\hline $\mathrm{L} 1=18500, \mathrm{~L} 2=26000 \mathrm{~m}$ & $\mathrm{k}=2 . \mathrm{E}-05 \mathrm{~m}^{-1}$ & $\mathrm{~L}_{\mathrm{cr}}=15300 \mathrm{~m}$ \\
\hline
\end{tabular}
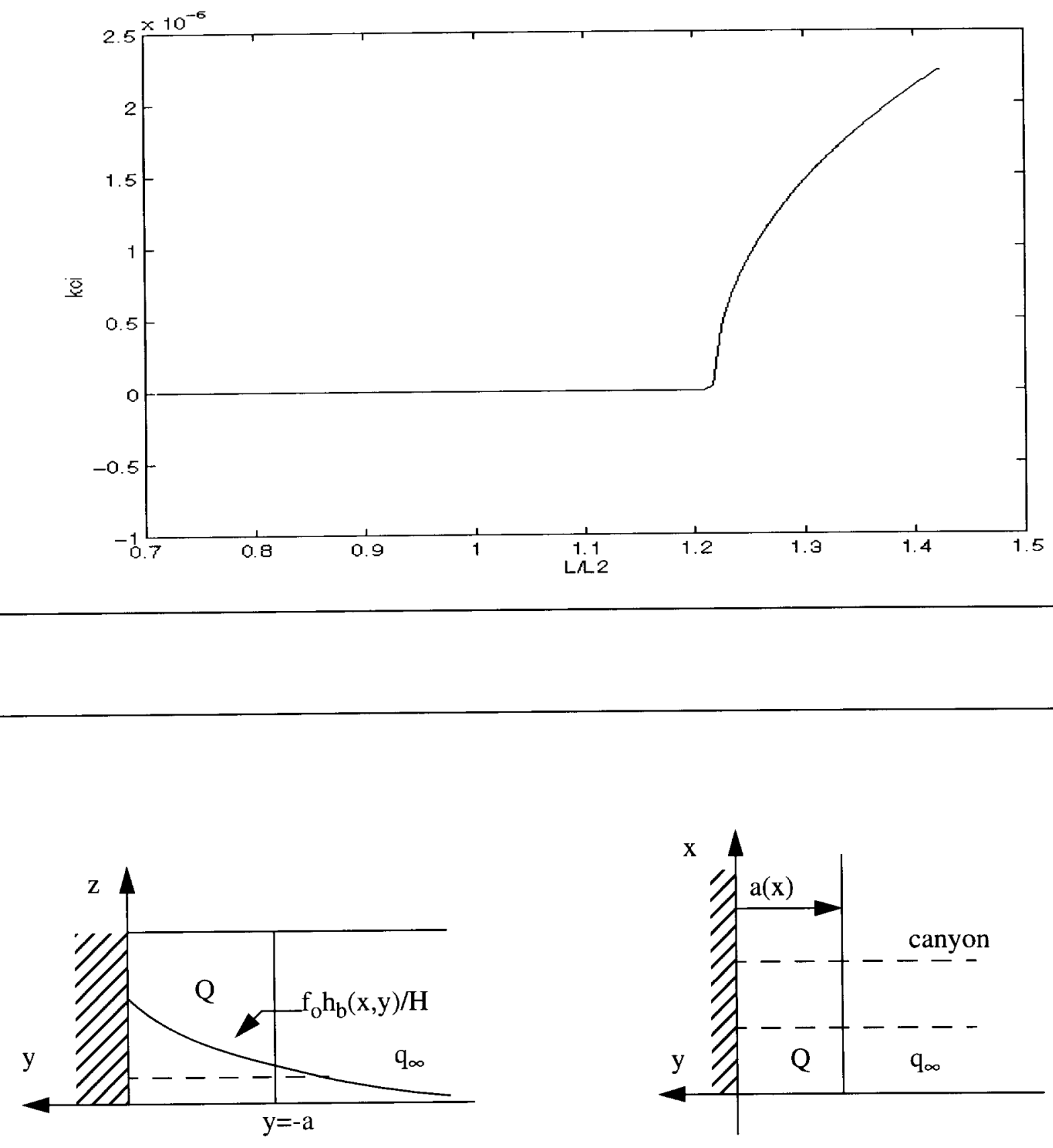

Figure 8: Time-evolution of an unstable jet (given by Fig. 5, with $b=1 / 2, s=0$ ) on a Gaussian canyon in a $2 \mathrm{D}$ contour surgery experiment. Dipole formation is observed. 


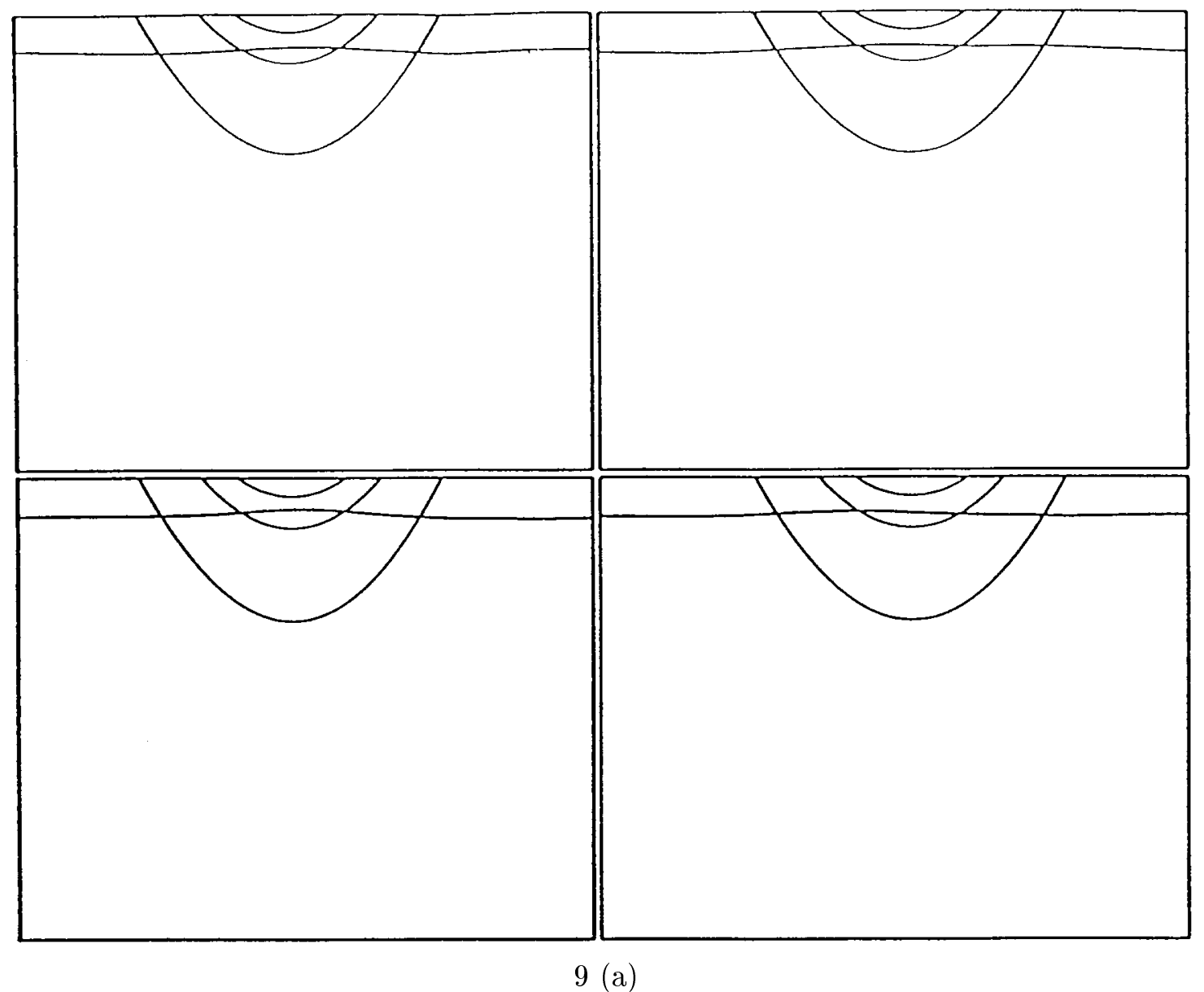

Figure 9: Time-evolution of an unstable jet (given by 3.1.B and 3.2) on a canyon with various width and depth: (a) shallow and wide canyon, stationary wave; (b) narrower canyon, amplifying and propagating waves; (c) narrow canyon, filament shedding; (d) deeper canyon, vortex generation and expulsion. 


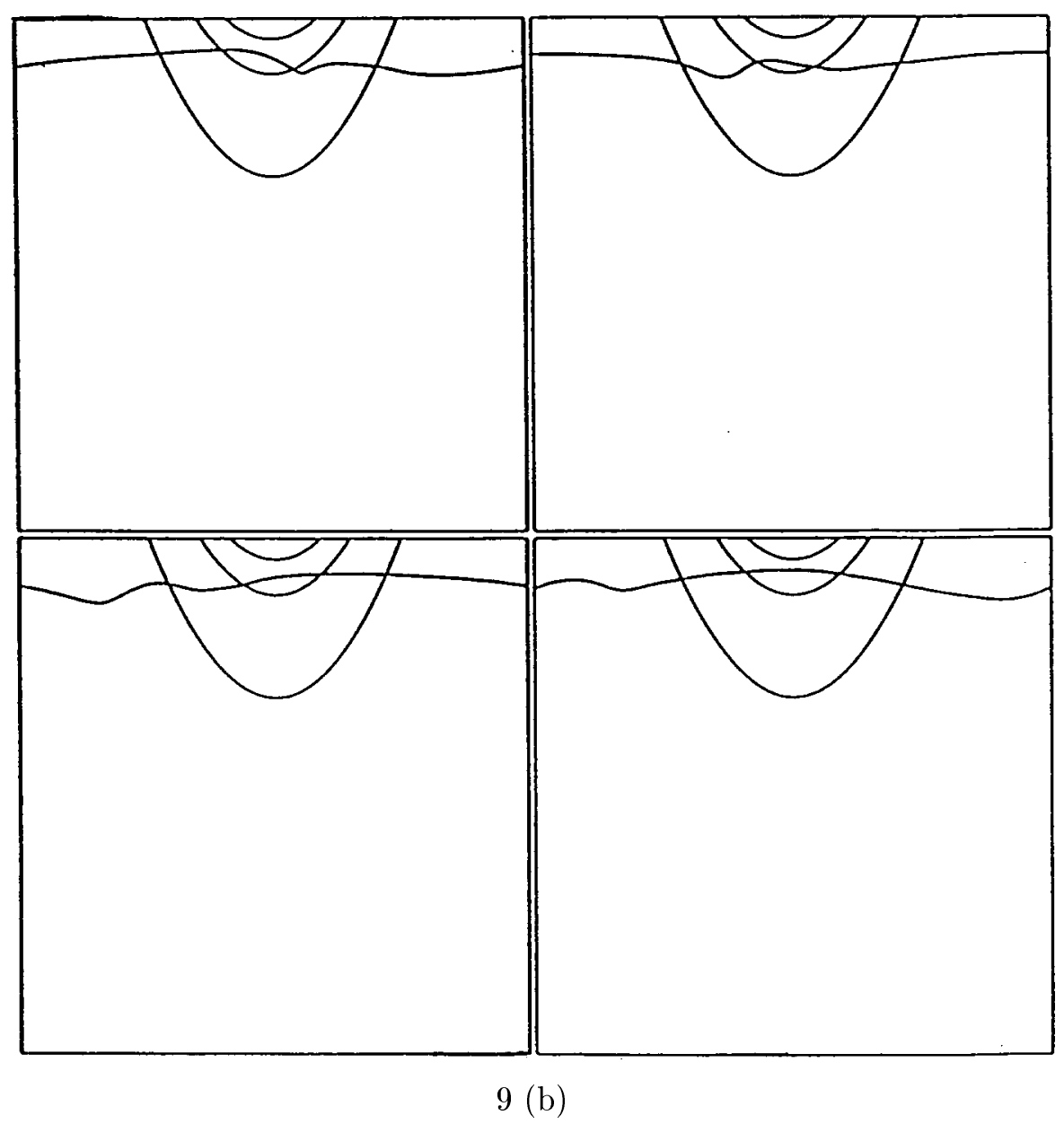

ESAIM: Proceedings, Vol. 1, 1996, pp. 481-501 


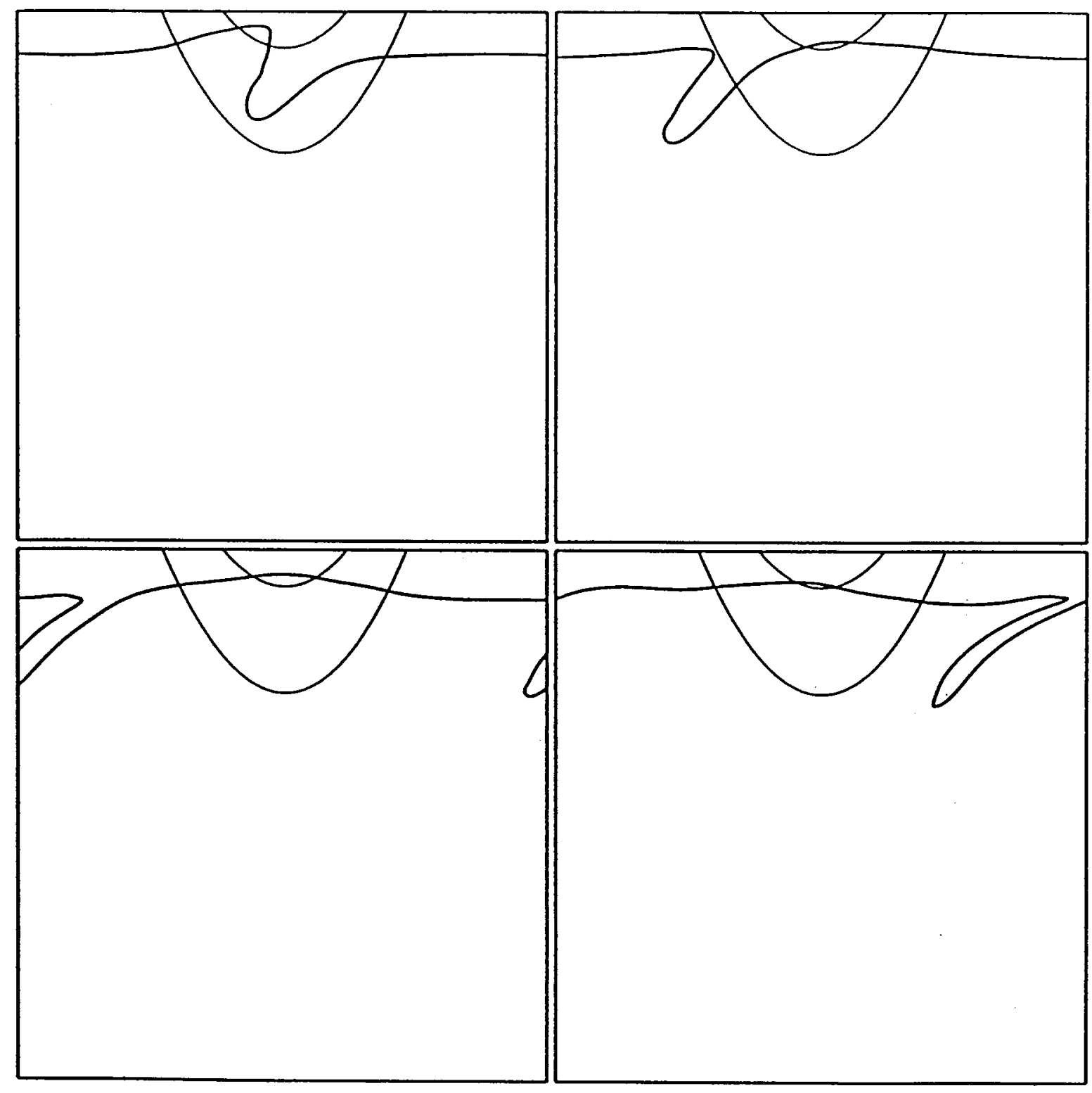

$9(\mathrm{c})$ 


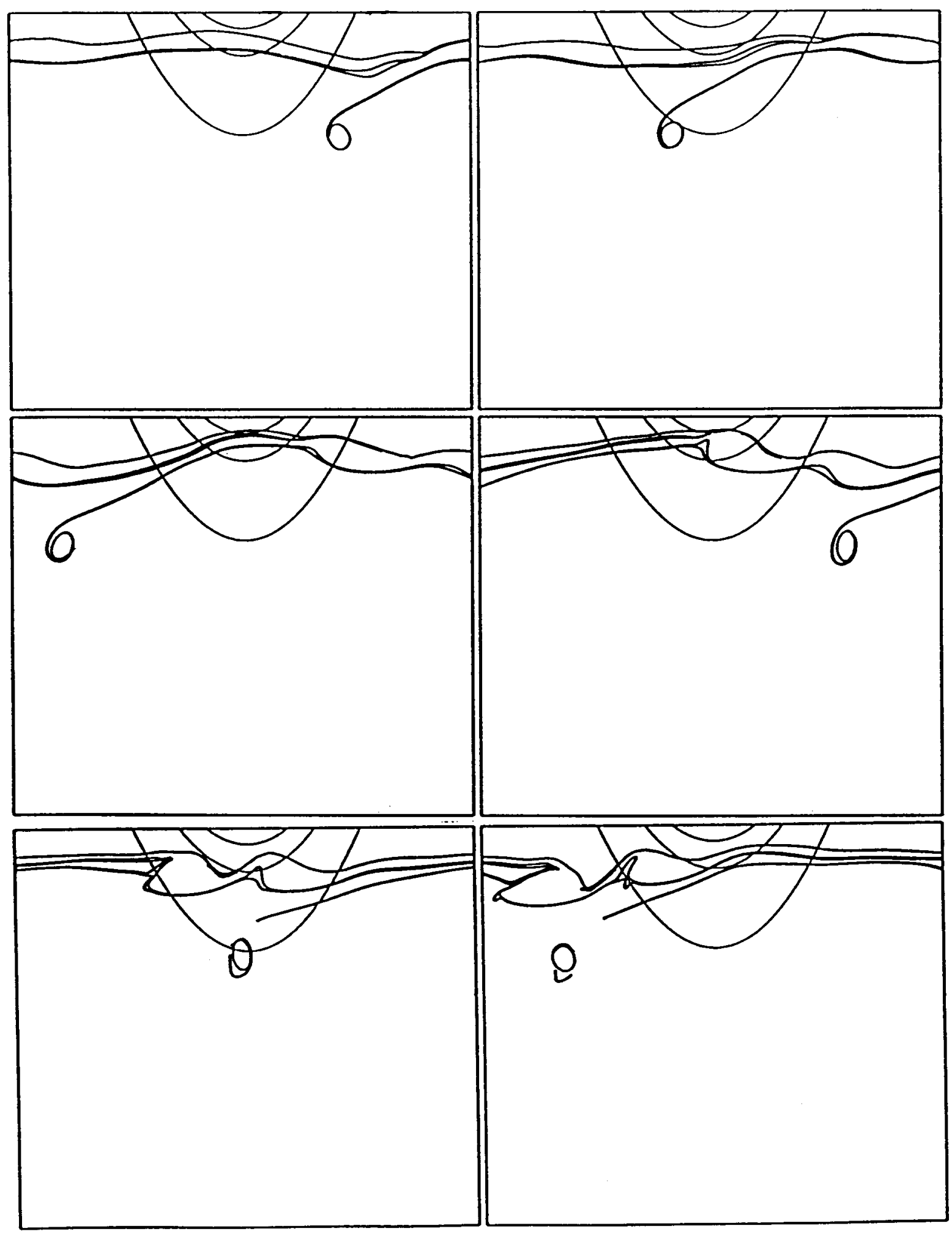

9 (d) 